\title{
Cardiac and Cerebral thrombosis in Behcet's Syndrome
}

\section{Freidoun Sabzi, Fahime ghasemi and Reza Faraji*}

Department of Cardiovascular Surgery, Imam Ali Heart Center, Kermanshah University of Medical Sciences, Kermanshah, Iran

\begin{abstract}
A 26 year old man without history of Behcet's syndrome was admitted to our department with a history of headache, fever, dyspnea, and weight loss. Transthoracic echocardiography and brain magnetic resonance imaging revealed a right atrial thrombus and sagital sinus thrombosis respectively. Surgery revealed the mass to be a thrombus. After 1 month of treatment with warfarin, he had experienced right atrial clot again. The von Willebrand factor antigen level was found to be elevated in blood examination. The large right atrial clot resected by second open heart surgery and medical treatment continued with cyclophosphamide, and corticosteroid, and thrombus did not reoccur in 6-month follow-up. To the best of our knowledge this is the first case of cerebral vein thrombosis associated with right atrial clot formation.
\end{abstract}

Keywords: Behcet's syndrome; Cardiac; Cerebral thrombosis; Sagittal sinus; Heart surgery

\section{Introduction}

Behcet's disease is an autoimmune and inflammatory disease of unknown etiology. It is a generalized disorder affects mainly young adults in Mediterranean, Middle Eastern, countries as Iran. It is defined by Mogulk as recurrent oral aphthous ulcers, genital ulcers, uveitis, and skin lesions [1,2]. As per Ames and colleagues Behcet's disease as a multisystem disorder may affect any organ in different combinations. Involvement of the heart is rare but combination of right atrium and sagital sinus thrombosis are exceptional. Careful medical literature review revealed no such case [3]. Other cardiac pathology that described by Ozkan et al. [4] and Goktekin et al. includes, aortic mitral valve prolepses, aortic root involvement, aortic valve regurgitation, coronary artery aneurysm, true myocardial aneurysm pericarditis, myocarditis, endocarditis, endomyocardial fibrosis, conduction system disturbances such as left or right bundle branch block or hemi block or complete heart block, coronary arteritis, acute myocardial infarction, and dilated cardiomyopathy [4-6]. Islim et al. [7] also found left ventricular diastolic dysfunction in echocardiography measurements.

\section{Case Report}

We report a patient who was admitted to the emergency unit of our cardiac surgery center. Patient did not have a history of the oral aphtous, or genitalia or skin ulcer. However, patient was found to have headache, fever, weight loss, and fatigue. Echocardiographic examination revealed a non-mobile mass attached to right atrium near coronary sinus ostium

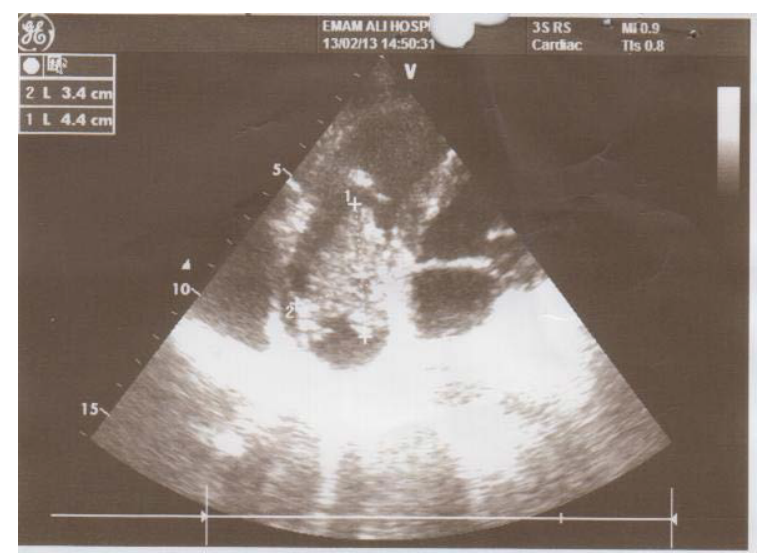

Figure 1: Echocardiography in four chamber view shows right atrial mass. that was initially thought to be an infected clot in unusual location, and the patient's condition was consulted with infectious disease specialists and neurologist. (Figure 1) Patient underwent surgery which revealed the mass to be a thrombus.

Brain MRI revealed sagital sinus thrombosis and patency of cerebral artery (Figure 2-4). After 4 weeks of anticoagulation with oral warfarin $(7.5 \mathrm{mg})$ daily and the target INR was 3.0 to 3.5 and antibiotics therapy, patient was readmitted to our center with the oral, genitalia and skin ulcers. Additionally, a right atrial thrombus was found again on echocardiographic examination. A careful reevaluation revealed pustular lesions on his skin and

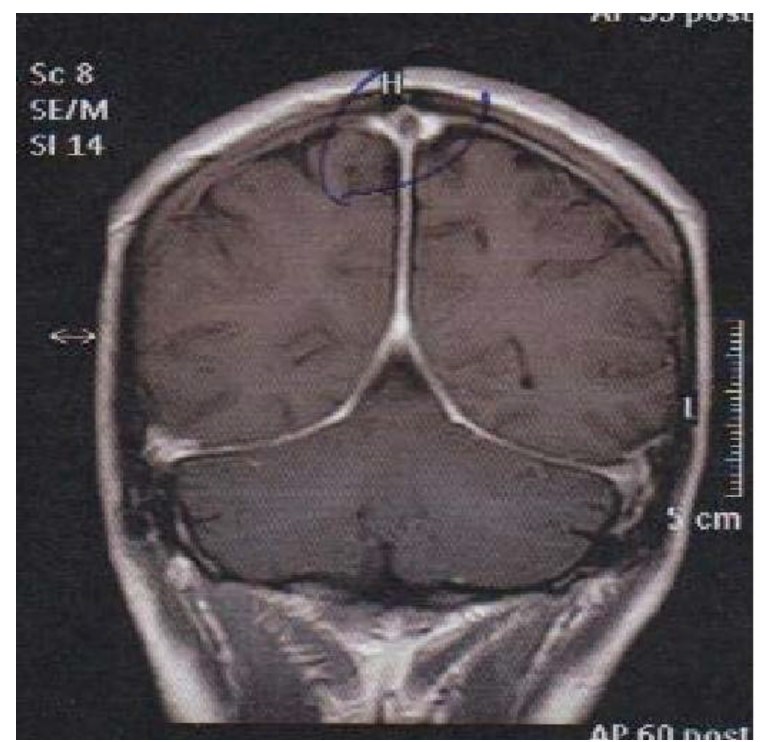

Figure 2: MRI revealed delta sign path gnomic of sagital vein thrombosis.

*Corresponding author: Sabzi F, Department of Cardiovascular Surgery, Imam Ali Heart Center, Kermanshah University of Medical Sciences, Kermanshah, Iran, Tel: +98 831 8360295; Fax: +98 831 9360043; E-mail: feridoun sabzi@yahoo.com

Received May 27, 2014; Accepted July 30, 2014; Published August 20, 2014

Citation: Sabzi F, Ghasemi F, Faraji R (2014) Cardiac and Cerebral thrombosis in Behcet's Syndrome. Gen Med (Los Angel) 2: 145. doi: 10.4172/23275146.1000145

Copyright: ( 2014 Sabzi F, et al. This is an open-access article distributed under the terms of the Creative Commons Attribution License, which permits unrestricted use, distribution, and reproduction in any medium, provided the original author and source are credited. 